\title{
Evolution of particle-scale dynamics in an aging clay suspension
}

\author{
R. Bandyopadhyay ${ }^{1}$, D. Liang ${ }^{1}$, H. Yardimci ${ }^{1}$, D. A. Sessoms ${ }^{2}$, M. \\ A. Borthwick ${ }^{3}$, S. G. J. Mochrie ${ }^{4}$, J. L. Harden ${ }^{2}$ and R. L. Leheny ${ }^{1}$ \\ ${ }^{1}$ Department of Physics and Astronomy, Johns Hopkins University, Baltimore, \\ MD 21218, USA. ${ }^{2}$ Department of Chemical and Biomolecular Engineering, \\ Johns Hopkins University, Baltimore, MD 21218, USA. ${ }^{3}$ Department of Physics, \\ Massachusetts Institute of Technology, Cambridge, MA 02139, \\ USA. ${ }^{4}$ Department of Physics, Yale University, New Haven, CT 06520, USA
}

(Dated: April 11, 2018)

\begin{abstract}
Multispeckle x-ray photon correlation spectroscopy was employed to characterize the slow dynamics of a colloidal suspension formed by highly-charged, nanometer-sized disks. At scattering wave vectors $q$ corresponding to interparticle length scales, the dynamic structure factor follows a form $f(q, t) \sim \exp \left[-(t / \tau)^{\beta}\right]$, where $\beta \approx 1.5$. The characteristic relaxation time $\tau$ increases with the sample age $t_{a}$ approximately as $\tau \sim t_{a}^{1.8}$ and decreases with $q$ approximately as $\tau \sim q^{-1}$. Such a compressed exponential decay with relaxation time that varies inversely with $q$ is consistent with recent models that describe the dynamics in disordered elastic media in terms of strain from random, local structural rearrangements. The amplitude of the measured decay in $f(q, t)$ varies with $q$ in a manner that implies caged particle motion at short times. The decrease in the range of this motion and an increase in suspension conductivity with increasing $t_{a}$ indicate a growth in the interparticle repulsion as the mechanism for internal stress development implied by the models.
\end{abstract}

PACS numbers: 82.70.Dd, 62.25.+g, 61.10.Eq

A signature feature of many disordered materials is the protracted evolution of their dynamic and thermodynamic properties. This process, known as aging, appears in a variety of systems including polymers [1], spin glasses [2], molecular glasses [3], and colloidal gels [4]. The observation of common behavior among seemingly disparate materials indicates generic underlying mechanisms for aging that have attracted strong theoretical interest into the nature of this out-of-equilibrium phenomenon [5, 6, 7, 8]. Experiments that probe aging have typically measured the temporal evolution of response functions, such as magnetic susceptibilities or elastic moduli, and far fewer experiments have characterized dynamical correlation functions in aging systems [4, 9, 10, 11]. However, because measurements of correlation functions generally access wave-vector dependence, they provide insight into variations in dynamics with length scale that can illuminate the microscopic origins of aging. To address this issue, we have conducted x-ray photon correlation spectroscopy (XPCS) studies on aqueous clay suspensions to characterize their intermediate scattering function during aging. The large wave vectors accessible with XPCS make these studies among the first to investigate the effects of aging on dynamics at interparticle length scales 12 .

The system under study is laponite XLG (Southern Clay Products), a synthetic Hectorite clay composed of discoidal particles $1 \mathrm{~nm}$ wide and $15 \mathrm{~nm}$ in radius. When dispersed in deionized water, the particles have a net negative charge leading to a repulsive colloidal suspension [13, 14]. At volume fractions $\phi \geq \phi^{*} \simeq 0.007$, laponite suspensions gradually transform into a soft solid with thixotropic response to stress [15] and anomalous rheology [13]. In addition, light scattering experiments have demonstrated that the long length-scale dynamics in such suspensions displays characteristic aging behavior [10, 11]. To study the evolution of particle-scale dynamics using XPCS, we prepared a suspension with $\phi=0.012$ by mixing oven-dried clay in deionized water. The resulting solution had $\mathrm{pH}=9.8$ and ionic strength $\simeq 2 \times 10^{-4}$ M. The clarified solution was filtered through $0.45 \mu \mathrm{m}$ pores to break up undissolved aggregates, and the age of the sample $t_{a}$ was measured from this filtering time.

Measurements were performed at sector 8-ID-I of the Advanced Photon Source (APS). Details regarding the beam line optics employed to create a partially coherent x-ray beam have been presented elsewhere 16]. The laponite suspension was contained in a sealed holder 700 $\mu \mathrm{m}$ thick with thin kapton windows for transmission scattering. The scattering intensity was recorded by a directilluminated CCD area detector (Princeton Instruments EEG model 37) $3.4 \mathrm{~m}$ after the sample covering a range of wave vectors $q$ from $0.05 \mathrm{~nm}^{-1}$ to $0.3 \mathrm{~nm}^{-1}$. At different sample ages a series of scattering images were recorded to determine the ensemble-averaged intensity autocorrelation function $g_{2}(q, t)=\frac{\left\langle I_{i j}\left(q, t_{a}\right) I_{i j}\left(q, t_{a}+t\right)\right\rangle_{i j}}{\left\langle I_{i j}\left(q, t_{a}\right)\right\rangle_{i j}\left\langle I_{i j}\left(q, t_{a}+t\right)\right\rangle_{i j}}$, where $\langle\ldots\rangle_{i j}$ denotes an average over pixels [16]. The minimum delay time $t$, set by the transfer rate of the CCD, was $1.6 \mathrm{~s}$. The longest time was set at $1000 \mathrm{~s}$ to avoid effects due to limitations in measurement stability that led to artificial decays in $g_{2}(q, t)$ at several thousand seconds. Measurements of $g_{2}(q, t)$ were made at ages from $t_{a}=1.3 \times 10^{4} \mathrm{~s}$ to $t_{a}=2 \times 10^{5} \mathrm{~s}$, during which time the sample evolved from a viscous liquid to a thixotropic solid capable of supporting its own weight.

Figure 1 shows results for $g_{2}(q, t)$ at $q=0.14 \mathrm{~nm}^{-1}$ measured at several $t_{a}$. The autocorrelation function displays a relaxation with an amplitude and character- 


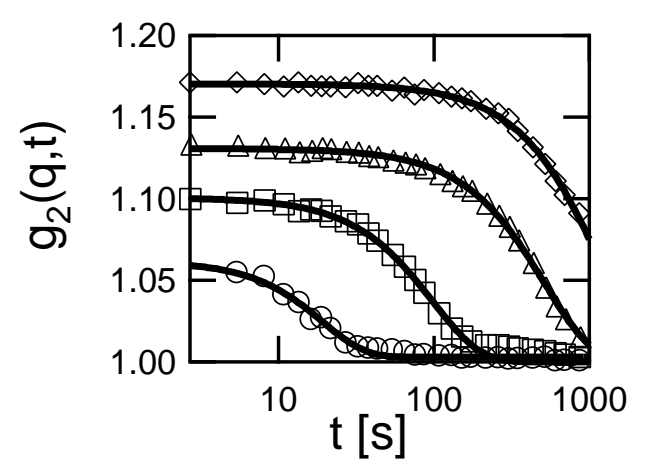

FIG. 1: Intensity autocorrelation function $g_{2}(q, t)$ at $q=0.14$ $\mathrm{nm}^{-1}$ for a laponite suspension of volume fraction $\phi=0.012$ at four ages: $t_{a}=1.3 \times 10^{4} \mathrm{~s}$ (circles), $3 \times 10^{4} \mathrm{~s}$ (squares), $9 \times 10^{4} \mathrm{~s}$ (triangles) and $2 \times 10^{5} \mathrm{~s}$ (diamonds). Solid lines are the results of fits to Eq. (1).

istic decay time that increase with increasing age. The dynamic structure factor $f(q, t)$ can be calculated from $g_{2}(q, t)$ using the Siegert relation. Modeling $f(q, t)$ with a stretched exponential lineshape $f(q, t)=A \exp (-t / \tau)^{\beta}$, the intensity autocorrelation function has the form

$$
g_{2}(q, t)=1+b\left[\operatorname{Aexp}\left[-(t / \tau)^{\beta}\right]\right]^{2}
$$

where $A$ is the short-time $(t<1 \mathrm{~s})$ plateau amplitude of $f(q, t), b$ is the Siegert factor, $\tau$ is a characteristic relaxation time, and $\beta$ is the stretching exponent that characterizes the lineshape. The solid lines in Fig. 1 are the results of fits to Eq. (1), which describes the data accurately over the full range of $q$ and $t_{a}$.

Results for the relaxation time $\tau$ and the amplitude of the decay in $g_{2}(q, t)$, given by $b A^{2}$, at $q=0.14 \mathrm{~nm}^{-1}$ are shown as a function of sample age in Figs. 2(a) and 2(b), respectively. Over the measured range in $t_{a}, \tau$ scales with age approximately as $\tau \sim t_{a}{ }^{\mu}$ with $\mu=1.8 \pm 0.2$. While such power-law scaling between $\tau$ and $t_{a}$ is a generic feature of aging systems, typically $\mu \leq 1$, and an exponent $\mu$ greater than one is unusual. Indeed, aging with $\mu=$ 1 has been observed with light scattering for a laponite suspension at $\phi=0.0136$ for $t_{a}>10^{4} \mathrm{~s}[10$. However, light scattering measurements observe a much stronger relation at early ages, specifically $\tau \sim \exp \left(t_{a}\right)$ [10, 11]. Conceivably, this rapid evolution of dynamics at early ages could extend to larger $t_{a}$ for smaller length scales, leading to an apparent scaling exponent of $\mu>1$. For the range of ages at which we observe $\mu>1$, however, $g_{2}(q, t)$ possesses a faster-than-exponential decay characteristic of solid-like behavior and thus does not support this picture. Instead, we interpret the observed scaling with $\mu>1$ as a potentially general feature of aging dynamics at interparticle length scales.

Specifically, the shape of $g_{2}(q, t)$ is inconsistent with a simple exponential relaxation $\beta=1$, except perhaps at the earliest age $t_{a}=1.3 \times 10^{4} \mathrm{~s}$, and best fits to Eq. (1)
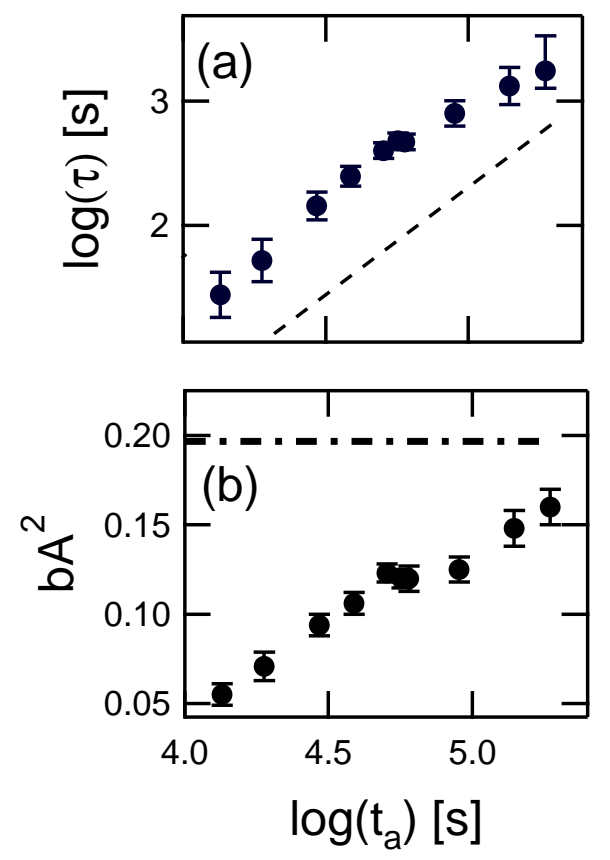

FIG. 2: (a) Characteristic relaxation time and (b) amplitude of $g_{2}(q, t)$ at short times for a laponite suspension of $\phi=0.012$ at $q=0.14 \mathrm{~nm}^{-1}$ as a function of age. The dashed line in (a) has a slope of 1.8. The dashed-dotted line in (b) shows the value of the contrast measured using a static aerogel sample.

give $\beta \approx 1.5$, with no systematic variation with age or $q$. Such a compressed exponential decay is incompatible with diffusive, fluid-like motion of the particles. While such hyperdiffusive relaxation with $\beta>1$ may seem unusual, similar behavior with $\beta=1.5$ has been observed at smaller wave vectors with dynamic light scattering on a number of soft solids including colloidal gels [4], clay suspensions [10], micellar polycrystals [9], and concentrated emulsions [9]. Recently, Cipelletti et al. [9], have advanced a microscopic picture for this dynamics, describing it in terms of the ballistic motion of elastic deformation in response to heterogeneous local stress. A specific model by Bouchaud and Pitard [17] associates local rearrangments, or "micro-collapses", of particles with the source for dipolar stress fields. A key feature of such dynamics is an inverse relation between wave vector and relaxation time, $\tau \sim q^{-1}$. As shown in Fig. 3, $\tau$ for the laponite suspension varies inversely with $q, \tau \sim q^{-0.9 \pm 0.1}$, over the range of wave vectors covered in the XPCS measurement in agreement with this prediction. This scaling holds for all $t_{a}$ where $\beta \approx 1.5$.

The observations with XPCS of $\beta \approx 1.5$ and $\tau \sim q^{-1}$ for laponite thus extends quite unexpectedly this solidlike dynamics into the particle-scale regime. In particular, combined with previous small angle dynamic light scattering measurements on colloidal gels at very small wave vectors [4], these results demonstrate that such hyperdiffusive relaxation can occur at length scales varying 


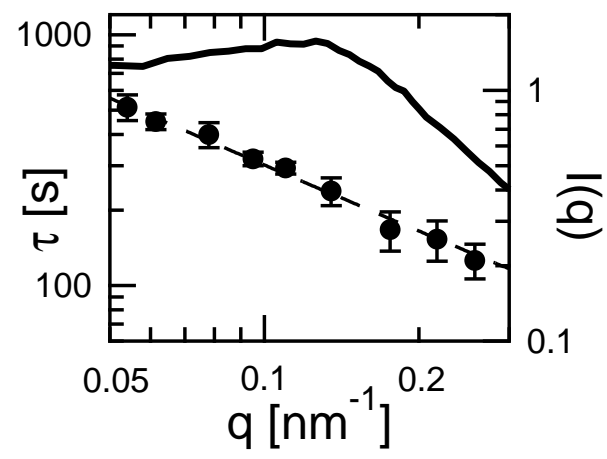

FIG. 3: Characteristic relaxation time $\tau$ (circles) at $t_{a}=$ $4 \times 10^{4} \mathrm{~s}$ and scattering intensity $I(q)$ (solid line) from SANS for a laponite suspension with $\phi=0.012$ as a function of wave vector $q$. The dashed line displays the fit result $\tau \sim q^{-0.9 \pm 0.1}$.

nearly five orders of magnitude relative to particle size. The relaxation mechanism suggested by Cipelletti et al., in which particles and their neighbors translate together with some velocity, provides a natural explanation for this broad range. In the absence of other dynamics, such motion leads to $\tau \sim q^{-1}$ for all $q$ greater than the inverse of the distance that particles travel under the strain.

We emphasize that the slow local dynamics of the laponite suspension contrasts with structural dynamics in glass-forming liquids. In glassy liquids, the main relaxation, known as the "alpha" relaxation, displays de Gennes narrowing at interparticle length scales such that $\tau q^{2}$ varies proportionally with the structure factor [18, 19]. We have investigated in detail the local structure in laponite suspensions through small angle neutron scattering (SANS) at the NIST Center for Neutron Research. The scattering intensity $I(q)$, also shown in Fig. 3 for a suspension of $\phi=0.012$, displays a weak interparticle structure factor peak near $q=0.13 \mathrm{~nm}^{-1}$ (corresponding to a center-to-center particle distance of $2 \pi / 0.13 \approx 48 \mathrm{~nm}$ for these $30 \mathrm{~nm}$ diameter disks). At higher $q, I(q)$ decays as $I(q) \sim q^{-2}$, characteristic of the disk form factor. Unlike with de Gennes narrowing, $\tau$ does not track the peak in $I(q)$. The insensitivity of $\tau$ to the interparticle correlations demonstrates conclusively that the slow dynamics of laponite are distinct from the collective diffusion of glassy systems.

While the picture that Cipelletti et al. introduce accounts well for these slow dynamics, an obvious question is the source of local stress in these soft solids. This issue is especially germane to laponite, since the suspensions solidify over the course of hours or days. Thus, unlike for systems quenched into a state far from equilibrium, laponite suspensions likely enter the solid phase annealed and free of residual stress. In particular, the microcollapse of particles [17] is unlikely in laponite for which the interparticle interactions are primarily repulsive at high $\mathrm{pH}$ and low ionic strength [13, 14, 20]. However, through the fast local dynamics implied by the XPCS results, we identify a microscopic mechanism, specifically the growth of the interparticle repulsion with $t_{a}$, by which stress can develop.

Information regarding the fast dynamics derives from the amplitude of $g_{2}(q, t)$ at short times. As shown in Fig. 2(b), this amplitude increases steadily with $t_{a}$. Ideally, $g_{2}(q, t) \rightarrow 2$ as $t \rightarrow 0$. Values less than two can reflect dynamic processes at times shorter than those experimentally accessible causing an apparent suppression in the structure factor amplitude, $A<1$. In addition, instrumental effects can lead to a Siegert factor $b<1$. To assess the instrumental effects, we compare the short time amplitude of $g_{2}(q, t)$ for laponite with that measured for a static aerogel sample. The amplitude of $g_{2}(q, t)$ for aerogel, shown by the dashed-dotted line in Fig. 2(b), gives the Siegert factor $b \simeq 0.20$, independent of $q$ and consistent with a value expected based on the x-ray optics. The suppression in the amplitude of $g_{2}(q, t)$ for the laponite suspension with respect to that of aerogel thus implies a partial decay to a plateau at inaccessibly short times and consequently to a two-step relaxation. Such two-step relaxations occur commonly in disordered systems including dense colloidal suspensions, polymer and colloidal gels, polymer solutions and supercooled liquids. In the case of supercooled liquids, the first part of this decay, termed the "beta" relaxation, represents the caged motion of a molecule in the confined space defined by its neighbors. Within this picture of caged motion, the short time plateau value is given by the Debye-Waller factor,

$$
A=\exp \left(-q^{2}\left\langle\bar{r}^{2}\right\rangle / 3\right) \text {. }
$$

Fig. 4(a) displays $\ln (A)$ versus $q^{2}$ for the laponite suspension at various $t_{a}$. The observed linear relationships support the picture of caged motion of the laponite particles at short times. Fig. 4(b), which shows values of the root mean squared displacements $\left\langle\bar{r}^{2}\right\rangle^{1 / 2}$ extracted from linear fits, reveals that this rapid motion becomes increasingly restricted spatially as the system ages.

We identify this increased restriction with an evolution in the interparticle potential. As mentioned above, the laponite become charged in aqueous solution, specifically via the unbinding of clay particles and subsequent dissociation of $\mathrm{Na}^{+}$ions from exposed faces, resulting in a screened Coulombic repulsion. To obtain evidence about this interaction, we determined the d. c. conductivity $\sigma$ of a suspension with $\phi=0.012$ as a function of $t_{a}$ by measuring the low-frequency impedance of a capacitor filled with the suspension. As shown in Fig. 4(b), $\sigma$ increases with increasing $t_{a}$. While the observed increase is modest, it is fully reproducible and far exceeds the uncertainty in the impedance measurements, which was roughly $0.1 \%$ 21]. Further, the relation between $\sigma$ and ionic concentration is highly nonlinear for low conductivity suspensions 22]. Hence, the small fractional increase in $\sigma$ implies a much larger change in ionic double-layer and, therefore, in repulsive interaction between particles.

An increasing repulsion not only explains the increas- 


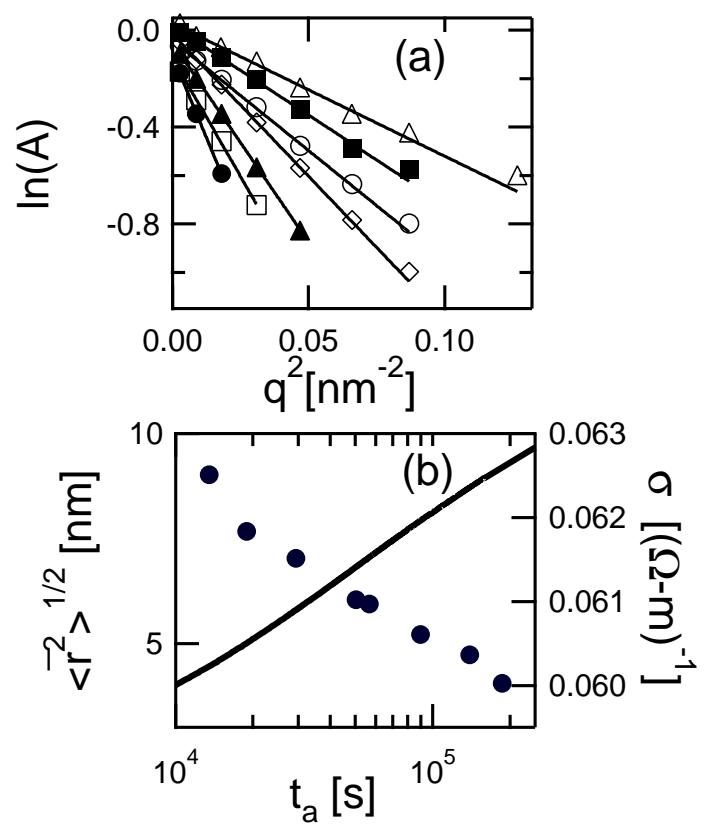

FIG. 4: (a) The natural log of the amplitude of $f(q, t)$ at short times $(t<1 \mathrm{~s})$ as a function of $q^{2}$ at ages $t_{a}=1.3 \times 10^{4} \mathrm{~s}$ (solid circles), $1.9 \times 10^{4} \mathrm{~s}$ (open squares), $3 \times 10^{4} \mathrm{~s}$ (solid triangles), $5 \times 10^{4} \mathrm{~s}$ (open diamonds), $9 \times 10^{4} \mathrm{~s}$ (open circles), $1.4 \times 10^{5}$ $\mathrm{s}$ (solid squares), and $2 \times 10^{5} \mathrm{~s}$ (open triangles). The solid lines show results of fits to Eq. (2). (b) The root mean square displacement $\left\langle\bar{r}^{2}\right\rangle^{1 / 2}$ (circles) for caged particle motion and d. c. conductivity $\sigma$ (line) for a laponite suspension of volume fraction $\phi=0.012$ as function of age. ing restriction in caged motion but also provides a mechanism for the slow dynamics. As the internal stress grows with increasing repulsion, it will surpass the local yield stress for mechanically weak arrangements of particles, leading to local restructuring and strain like that pictured by Cipelletti et al. This connection between fast and slow local dynamics in laponite thus demonstrates how aging does not always require quenched disorder but rather can occur via the build-up of stress in an otherwise annealed system. Finally, we note that while the interactions were predominantly repulsive for the solution conditions we employed [14, 20], laponite can form attractive fractal aggregates under many conditions, such as with added salt [23, 24]. The amplitude of fast dynamics in such aggregates should vary as $\left\langle\bar{r}^{2}\right\rangle \sim 1 / \kappa_{0}$, where $\kappa_{0}$ is the spring constant of the attractive bond 25]. Increasing these particle attractions would decrease $\left\langle\bar{r}^{2}\right\rangle$ and build internal stress, possibly leading to a scenario that mirrors our observations for the repulsive system. This correspondence between attractive and repulsive suspensions thus illustrates the potentially broad relevance of this mechanism for aging, which relies not specifically on disorder but rather on evolving interparticle interactions.

We thank B. Chung, D. Ho, S. Narayanan, and A. Sandy for their assistance. Funding was provided by the NSF (DMR-0134377 and DMR-0071755). Acknowledgement is also made to the donors of The Petroleum Research Fund, administered by the ACS. Use of the APS was supported by the DOE, Office of Basic Energy Sciences, under Contract No. W-31-109-Eng-38.
[1] L. C. E. Struik, Physical Aging in Amorphous Polymers and Other Materials (Elsevier, Amsterdam, 1978).

[2] P. Norblad, in Dynamical properties of unconventional magnetic systems, edited by A. T. Skjeltorp and D. Sherrington (Kluwer, Boston, 1998).

[3] H. Yardimci and R. L. Leheny, Europhys. Lett. 62, 203 (2003).

[4] L. Cipelletti, et al., Phys. Rev. Lett. 84, 2275 (2000).

[5] L. F. Cugliandolo, J. Kurchan and L. Peliti, Phys. Rev. E 55, 3898 (1997).

[6] J.-P. Bouchaud, in Soft and Fragile Matter: Nonequilibrium Dynamics, Metastability and Flow, edited by M. E. Cates and M. R. Evans (IOP, Bristol, 2000).

[7] S. Franz et al., Phys. Rev. Lett. 81, 1758 (1998).

[8] S. M. Fielding, P. Sollich, and M. E. Cates, J. Rheol. 44, 323 (2000).

[9] L. Cipelletti, et al., Faraday Discuss. 123, 237 (2003).

[10] M. Bellour et al., Phys. Rev. E 67, 031405 (2003).

[11] B. Abou, D. Bonn and J. Meunier, Phys. Rev. E 64, 021510 (2001).

[12] R. E. Courtland and E. R. Weeks, J. Phys.: Condens. Matter 15, S359 (2003).

[13] A. Mourchid et al., Langmuir 11, 1942 (1995).
[14] S. Bhatia, J. Barker and A. Mourchid, Langmuir 19, 532 (2003)

[15] N. Willebacher, J. Colloid Interface Sci. 182, 501 (1996).

[16] D. Lumma et al., Phys. Rev. E 62, 8258 (2000).

[17] J.-P. Bouchaud and E. Pitard, Eur. Phys. J. E 6, 231 (2001).

[18] P. N. Segrè and P. N. Pusey, Phys. Rev. Lett. 77, 771 (1996).

[19] A. Tölle et al., Eur. Phys. J. B 5, 231 (1998).

[20] M. Morvan et al., Colloids Surf. A 82, 193 (1994).

[21] Converting from impedance to $\sigma$ required scaling by a multiplicative constant that had roughly $5 \%$ uncertainty, based on the capacitor geometry, but did not introduce error in the variation of $\sigma$ with $t_{a}$.

[22] R. W. O'Brien and W. T. Perrins, J. Colloid Interface Sci. 99, 20 (1984); R. Kan and P. N. Sen, J. Chem. Phys. 86, 5748 (1987).

[23] T. Nicolai and S. Cocard, Langmuir 16, 8189 (2000).

[24] F. Pignon, J.-M. Piau and A. Magnin, Phys. Rev. Lett. 76, 4857 (1996).

[25] A. H. Krall and D. A. Weitz, Phys. Rev. Lett. 80, 778 (1998). 